\title{
Service-Oriented Device Integration for Ubiquitous Ambient Assisted Living Environments
}

\author{
Javier Andréu Pérez ${ }^{1}$, Juan Antonio Álvarez², \\ Alejandro Fernández-Montes ${ }^{2}$, and Juan Antonio Ortega ${ }^{2}$ \\ ${ }^{1}$ Department of Communication System, InfoLab21, Lancaster University, \\ Lancaster, United Kingdom \\ j.andreu@lancaster.ac.uk \\ ${ }^{2}$ Department of Computer Languages and Systems, University of Seville, Seville, Spain \\ jaalvarez@us.es, afdez@us.es, jortega@us.es
}

\begin{abstract}
As a result of the increment of population in countries of Europe, a lot of efforts from European Authorities are coming from. In our research we want to bring forward a suite of developments related to build a ubiquitous AAL (Ambient Assisted Living) environment. We consider that recent approaches are based on ad-hoc technologies so its application is in this context isolated just in one domain of application. Our approach addresses to a reliable services platform for heterogeneous devices integration. On this basis we want to consider as well, the underlying benefits that a Service-oriented platform is giving to us in our Ambient Assisted Living Applications.
\end{abstract}

Keywords: Ambient Assisted Living, Services Oriented Architectures.

\section{Introduction}

Nowadays Ambient Assisted Living environments are composed by different kinds of devices such as mobile phones, embedded devices, and wired or wireless sensors. Although nowadays are raising some efforts based on communication standards for telemedicine devices but its adoption is going slow. In addition, both biomedical and users devices often runs on different network protocols which obstruct a real integration between devices.

Smart sensors have been a breakthrough in the application of monitoring mobile objects or individuals. On this basis Smart Sensors are becoming a main "actor" on AAL environments. Thanks to nanotechnology provided by MEMS, in the recent years has been able to embed new media hardware, such as brand new wireless technologies. Nowadays, with the increase in memory and processing on devices is possible to install new development tools for instance web services, databases and artificial intelligence techniques.

On the basis of these new trends we decided to include the latest technology of Service-Orientation on devices in an AAL environment. On the one side is DPWS (Device Profile for Web Services) which has been successfully put into both industrial automation and home systems and on the other side, OSGi (Open Services 
Gateway initiative) specifications, which are used in applications such as mobile phones, automobiles, industrial automation, building automation, grid computing.

The following paper is organized as follows: Firstly in section 2 we are going to explain the why and wherefores of this services approach for devices integration; in section 3 the benefits of this approach regarding AAL; in section 4 how the approach is carry out; and finally in 5 this platform performing in an AAL scenario.

\section{A Services-Oriented Platform for Ubiquitous Computing}

In most cases, caring for elderly people requires close monitoring of their daily activities. It gives rise to limit their privacy, whilst at the same time it brings about an excessive workload for caregivers. Ubiquitous computing as a real health assistant is being one of the most prominent approaches in this area. As a result, caregivers can monitor real-time health data and behavior of elderly people. It is accomplished using a network of sensors placed at strategic points in a living environment of elderly people as well as objects of everyday use [1]. However we are concerned about all this works are based on ad-hoc technologies. They always consider just for these targets a same kind of both devices and network protocols.

\subsection{Experiences of Services-Orientation for AAL}

Working from the principles above remarked, we are going to analyze in detail the most important benefits which it offers us in the development of an Ambient Assisted Living scenario compound of several devices.

Currently, targets of sensor networks are to create an ambient intelligence environment; therefore we can use it to develop a platform to attain a reactive system in this context. There are several works which have studied this solution and some of them tried to make up an ambient intelligent platform considering the AAL requirements [2]. In this line, our latter research [3] discussed the development, design, characterization and tests of miniaturized wireless and wearable sensors both ECG and Fall Detector. In this research we describe how a wireless sensor platform is incorporated with the TAAL (Telecare Ambient Assisted Living). This architecture aims to optimize accurate data delivery onto a WSN healthcare environment defining three kinds of roles. Thus this approach clarifies the development of an Ambient Assisted Living using wireless sensor network. We defined three roles that identified the different behaviors between motes. Although, the nodes of TAAL were too much related to IEEE 802.15.4, this abstraction is still quite efficient, really constructive and correct because every mote has only a suite of several responsibilities which provide analysts and programmers minimal necessary abstraction to carry out the design and development of AAL platforms.

In previous approach [4] we have also developed an assisted living environment using RFID technology combined with speech techniques, zigbee devices, analog ADXL203 accelerometers, and PDAs. All of these for creating different and independent services such as location, audio-reminder and communication services. 


\subsection{Why Services-Orientation?}

Similar to the situation with the traditional distributed computing, a ubiquitous environment also needs to deal with resources in a transparent, open, and scalable manner; accordingly a new level of abstraction is required. With the Service Oriented Abstraction, developers realized that reuse and maintaining code do not remain a complex task. In the business world SOA has been accepted as a mature technology that solves the problems of interoperability between technologies [4] and the ability to add functionality without having to redo systems by exploiting the collaboration of services.

Based on this approach one highlight is the ease of integration between communication protocols, for example IP-based networks with wireless sensor networks, which are frequently used in ambient intelligence applications. This integration by means of a service abstraction provides us the capacity to deal with issues from high level layers which places us in a better position since we are able to develop versatile applications.

Services-Orientation is being applied in several domains successfully. Nowadays, its application is arising interesting results in other domains such as ubiquitous computing. However, there is a general interest to deploy this new paradigm in devices with limited computational resources. In this way, developers of ambient intelligent systems are getting the drift of what are the advantages to use a services abstraction.

Services Principles. As we remarked above, with services oriented architecture we were also able to solve some common issues of WSN. We are going to clarify this assertion firstly, by putting in the center the SOA paradigm. On this basis all developments must accomplish with this principles in the concept of "Service" in order to be considered a reliable Service Oriented Architecture. In [5] the prominent methodologist of the Services Abstraction, Thomas Erl, defined this list of principles as structural requirements in order to establish the SOA system design: Reusability, Formal Contract, Low Coupling, Composability, Low Coupling, Discoverability, Stateless, Autonomy and Scalability.

\section{Our Solution in AAL Environments}

In this section we are going to present our development and resources in order to create a services-oriented platform for AAL, describing initially the technologies used and afterwards an integration scenario.

\subsection{Description of Used Technology}

Used technologies in our work. We used Device Profile for Web Services (DPWS) and OSGi (Open Services Gateway Initiative), with the purpose of deploying reliable services orientation on devices in an Ambient Assisted Living scenario. In the following paragraphs we are going to give a description on it.

W3C Web Services as a basis. A new tool that fits very well with our needs is a new version of the protocol for UPnP devices; this is DPWS, which is based on well known protocols and several Web service specifications. The main objective DPWS 
is to establish for resource-constrained devices the same architecture which is used for services on the web.

DPWS specifies further mechanisms services discovery (WS-Discovery). It provides events subscription (WS-Eventing), online information about services and devices (WS-Metadata), definition of security policies and behavior, (WS-Policy), communication model based on semantic rules (WS-Addressing) [6].

$O S G i$ is a framework which provides to developers an integrated development environment based on $b$ (also named bundles). This environment offers several useful APIs for the governance of a system composed by services as a whole. Some prominent parts of the framework are the management services API (ServiceRegistration, ServiceTracker and ServiceReference) and Service Lifecycle Management API. [7]

DPWS-OSGi Bridge is really a framework of integration between these previous technologies. DPWS and OSGi are complementary for an easy application development [8]. On this basis, we developed a DPWS framework using Java CDC foundation profile and integrated into the OSGi framework by "bundelizing" its component model, i.e disaggregating its dependencies, both third party libraries and DPWS resources; between clients and servers in separated bundles but taking into account its dynamic and distributed performance. Therefore, we had to develop a shared bundle which contains and supplies in real time all of these dependencies for each one of the sides (clients and services).

\subsection{Integrating Ubiquitous Devices in AAL Environments}

An ubiquitous AAL scenario is composed of various remote network devices in different networks. Depending on who is monitored, we can find PAN Personal Area Network (PAN) focusing on individuals or a LAN or WAN network, depending on the scope of monitoring. In terms of intelligent environments and ubiquitous computing the main objective is to fulfil the terms of pervasive ubiquity, so we need to consider a complete mobility of users. As a result of recent technological advances and remote environments, we consider wireless technology is the best for PAN networks so far. Therefore in our approach for an AAL architecture based on DPWS we have taken into account the possibilities of communication between WSN and other networks. In Fig. 1 is depicted a scenario of interaction between different networks.

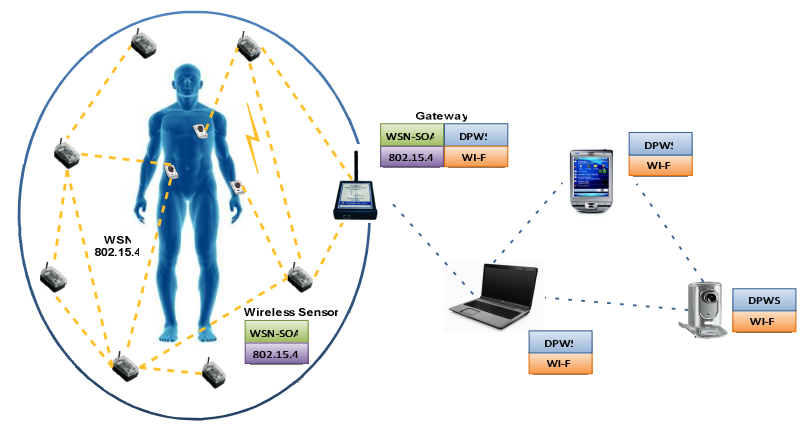

Fig. 1. This figure represents an outline of a patient monitoring through a network of sensors and devices with which it interacts 
On the one side it is the WPAN (Wireless PAN) and on the top of it, we enabled a service discovery, multicast invocation, event and subscription from a special lightweight SOA for WSN (WSN-SOA). We realized that the use of W3C standards for web services architectures are computationally complex, in addition to the use of XML files is an issue for an efficient transmission in wireless sensor networks. A Binary XML is very verbose because of its textual heritage and it has many rules for friendliness toward internationalized text. For wireless sensor networks we determined that ad-hoc architecture of services for this type of sensors is the best solution. Technological restrictions on energy consumption forced us to perform services implementations in accordance with the instruction and libraries provided by the manufacturer.

On the other side, we have IP-Based devices; onto these we deployed directly a java DPWS stack enabling directly service-oriented Communication. DPWS defines a distributed service-oriented architecture above web-oriented protocols. The benefits of DPWS on these devices enable us the same features of the ad-hoc implementation WSN-SOA. That being said, in this case we established the standard protocols for Web Services (SOAP, WSDL) plus some underlying protocols such as WSManagement, which provides us management of deployed services through a graphical command control unit, WS-Policy, which describes the characteristics, capabilities and requirements for performance and interoperability and finally WS-Metadata, which provides us new information about end-points (the entity referenced with a service that can be identified) in order to interact with another services.

As we show in Fig. 1, we need to provide a bridge between the two technologies. This bridge provides us the translation of services, data aggregation, and discovery. Additionally it abstracts the WSN as if it were a separate device inside the IP-Based network. As such, OSGi defines service oriented architecture in a component model on a local network node. The abstraction across OSGi bundles provides us the ability to take mirrored in the bridge each one of realistic services from devices. This abstraction hides the complexity involved in the heterogeneity, distribution and modularity from developers and provides integration into the OSGi context.

In ubiquitous systems is important to offer some kind of intelligence. For Telecare applications are important to achieve a quick diagnosis of the information processed by the sensors. That means that information derived from sensors should be digested in an intelligent manner, for example using reasoning engines. It is therefore necessary to add new applications in the system with the purpose of offering this layer of intelligence. The architecture defined before allows us the capacity to offer distributed applications through DPWS, and in turn, we are able to integrate reasoning engines with another services in the OSGi platform. In our research we considered the requirement to provide reasoning engines as another services. For example, different patients do not have the same heart rate or do not have the same flexibility of movement. Therefore the use of intelligent adaptive techniques in the system is essential [9].

\section{Ambient Assisted Living Scenario}

In this section, we will explain a set-up of the technological platform, according to the composition of an assisted living scenario. 
Peter is involved in an Ambient Assisted environment in his home with a wireless network monitoring his motion activity, using a 3D accelerometer, a 3D compass, and pedometer, on the other side two IP network devices: a web camera and a PDA, and finally a Bluetooth mobile phone. His PDA polls each minute the gathered data from Zigbee devices. They are extracting statistical values from the patient (mean, variance, median, direction, kurtosis, $25 \%$ percentile and $75 \%$ percentile), and all of this information will be send it by a sms message to his relatives.

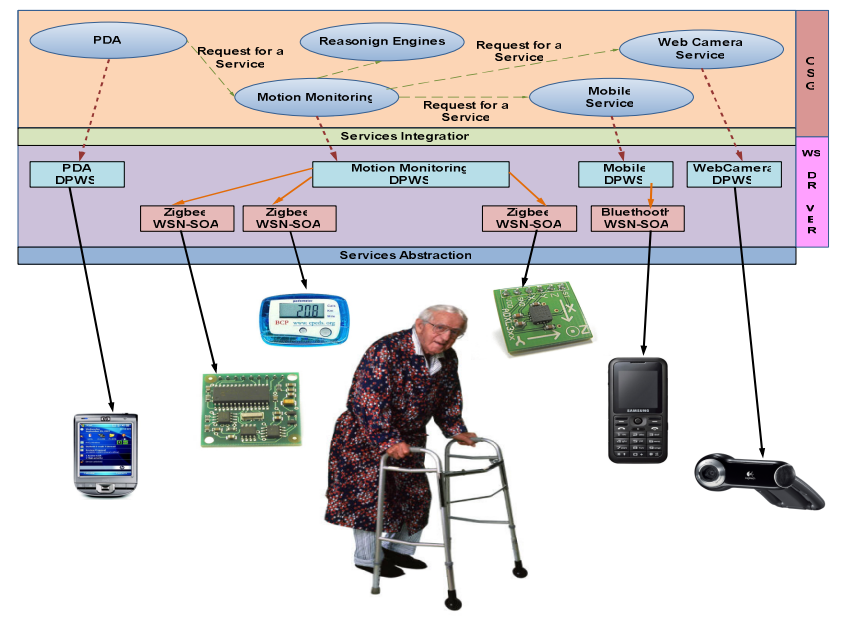

Fig. 2. Peter's Assisted Living Scenario explained by layers of abstraction

A fundamental problem in an AAL scenario like that is the integration of different technologies in order to achieve a complete performance of the required scenario. For this reason, our next step will be to create abstractions to provide us a proper objectoriented architecture. For the development of our platform we used the Java programming language, based on the specification for mobile devices J2ME (Java 2 Micro Edition). For example in the context of Bluetooth and 802.11 we made a release of WSN-SOA based on the Java standards JSR-82 and JSR-185 respectively. At any rate, as in figure 2 shows, DPWS can also be deployed directly in a straightforward manner on devices, for example onto IP-Based devices such as PDAs or the webcams. WSN-specific services can be grouped into the DPWS in an independent suite of services for full integration. This suite can be customized to perform the requirements of this scenario. In the case of the figure, we decided to group all the parameters for monitoring patient's movements in a DPWS service. Instead, the Bluetooth mobile is a separate service. Once we have made out basically WS-Drivers for each one of devices, we turn to a top layer of integration. In the upper layer we integrate the devices in an OSGi platform, creating a suitable framework for developers of AAL applications. Developers will be able to format merely their AAL scenarios in accord with their requirements. OSGI generic API provides us a first transparency level for develop both DPWS clients and services and it is what give rise to mask all the discovery, description and control protocol details to developers. In addition it is 
possible to add onto the peter's enviroment new additional distributed tools like reasoning engines for digest stream data from sensors, or even data repositories which are offered by means of OSGi bundles as is shown in the figure.

\title{
5 Conclusion and Future Work
}

In this section we are going to bring forward advantages of our work and finally a brief description of our technological approach across our future works and conclusion.

\subsection{Main Advantages of AAL Environments}

Event-Driven Services. A patient could be monitored continuously to detect a medical disorder for example ECG and $\mathrm{SpO} 2$. In this case we need to track data from burst signals and blood glucose and temperature, which are directly captured in only one measurement. An event driven architecture enables exploit opportunities more quickly and respond more timely to events like heart conditions. For example after a critical cardiac episode, it is very important to detect the problem as quickly as possible, because the chances of successful recovery of the patient decrease an hour after the heart attack (golden hour).

Energy Awareness. No system of ambient intelligence is feasible if there is not a good management of energy consumption. In order to save energy we can vindicate the principle of autonomy and low-coupling in the performance of each service, in doing so, we may suggest that this programming environment is appropriate for an event-drive execution model which is a reliable approach to save energy. Each of the services operates from time to time only when it is required precisely and only to execute its operation or functionality defined.

\begin{abstract}
Nowadays a complete ubiquitous ambient assisted living environment is integrated by several kinds of different devices. On a fundamental level, sometimes for some kinds of applications it would be necessary to combine knowledge from different sources, for instance RFID and smart sensors [10]. These kinds of sources might have different either system architectures or programming language. This principle emphasizes to hide as much as possible the underlying details of a service. So doing directly it enables and preserves the previously described low coupling principle.
\end{abstract}

\subsection{Summary}

In this paper, we showed a software architecture based on SOA for devices that uses as two layers of abstraction based on the combination of DPWS and OSGi. To achieve a scalable platform for our AAL prototypes has been developed a universal integration platform described above, with the aim to fulfill the basics service principles. But recent technological developments such as $3 \mathrm{G}$ or WiMAX ahead, force us to develop new WSN-SOA optimized implementations for these new breakthrough technologies as well. However, the platform due to its modular design and layering is optimized for these new changes. Thereby we can ensure the integration of brand new devices for remote care, on this basis we are currently running a project based on the 
integration of Google Android by means of Luminis OSGi. Additionally we are going about the integration of new intelligent self-developing and self-adaptative techniques in order to achieve a fully automatic customization for the monitoring state of patients. These new reasoning engines will be offered directly on the same platform explained in this article.

Acknowledgments. The work has been partly supported by the next projects: FAMENET-InCare (TSI2006-13390-C02-02), CUBICO (TIC2141) and OSAMI (ITEA 2 ip07019, TSI-020400-2008-114).

\section{References}

1. Dengler, S., Abdalkarim, A., Falko, D.: Sensor/Actuator Networks in Smart Homes for Supporting Elderly and Handicapped People. In: Proceedings of the 21st International Conference AINAW, pp. 863-868 (2007)

2. Bottaro, A., Simon, E., Seyvoz, S., Gérodolle, A.: Dynamic Web Services on a Home Service Platform. In: 22nd International Conference on Advanced Information Networking, pp. 378-385 (2008)

3. Andreu, J., Viudez, J., Holgado, J.A.: An ambient assisted-living architecture based on wireless sensor networks. In: 3rd Symposium of Ubiquitous Computing and Ambient Intelligence, pp. 239-248 (2008)

4. IBM Press: The New Language of Business SOA. Bulletin (Feburary 2007)

5. Erl, T.: SOA principles of service design. Prentice Hall, Englewood Cliffs (2007)

6. Device Profile for Web Services Specification. Microsoft Corporation (2006)

7. Open Services Gateway Initiative Specification Overview, http: / /www.osgi.org/resources/spec_overview.asp

8. Wen-Wei, L., Yu-Hsiang, S.: Using OSGi UPnP and Zigbee to Provide a Wireless. In: Ubiquitous Home Healthcare Environment, Ubicomm 2008, pp. 268-273 (2008)

9. Angelov, P., Zhou, X.: On line learning fuzzy rule-based system structure from data streams. In: Fuzzy Systems Proceedings, pp. 29-35 (2006)

10. Álvarez, J.A., Pérez, C., Angulo, C., Ortega, J.A.: Combining Smart Tags and Body Fixed Sensors for Disabled People Assistance. In: Knowledge-Based Intelligent Information and Engineering Systems, pp. 10-17 (2007) 